\title{
A simulation approach to the process planning problem using a modified particle swarm optimization
}

\author{
Wang, J.F. ${ }^{\text {a, }}{ }^{*}$, Kang, W.L. ${ }^{a}$, Zhao, J.L. ${ }^{a}$, Chu, K.Y. ${ }^{a}$ \\ ${ }^{\mathrm{a}}$ Department of Mechanical Engineering, North China Electric Power University, Baoding, China
}

\begin{abstract}
A B S T R A C T
Due to the complexity and variety of practical manufacturing conditions, computer-aided process planning (CAPP) systems have become increasingly important in the modern production system. In CAPP, the process planning (PP) problem involves two tasks: operation determining and operation sequencing. To optimize the process plans generated from complex parts, the traditional particle swarm optimization (PSO) algorithm is modified. Efficient encoding and decoding population initialization methods have been developed to adapt the PP problem for the PSO approach. In addition, to avoid the proposed approach becoming trapped in local convergences and achieving local optimal solutions, parameters are set to control the iterations. Several extended operators for the different parts of the particles have been incorporated into the traditional PSO. Simulation experiments have been run to evaluate and verify the effectiveness of the modified PSO approach. The simulation results indicate that the PP problem can be more effectively solved by the proposed PSO approach than other approaches.
\end{abstract}

\begin{tabular}{l} 
A R T I C L E I N F O \\
\hline Keywords: \\
Process planning \\
Operation determining \\
Operation sequencing \\
Particle swarm optimization \\
Extended operator \\
*Corresponding author: \\
wjf266@163.com \\
(Wang, J.F.) \\
Article history: \\
Received 29 January 2016 \\
Revised 27 March 2016 \\
Accepted 10 May 2016
\end{tabular}

(c) 2016 PEI, University of Maribor. All rights reserved.

\section{References}

[1] Zhang, F., Zhang, Y.F., Nee, A.Y.C. (1997). Using genetic algorithms in process planning for job shop machining, IEEE Transactions on Evolutionary Computation, Vol. 1, No. 4, 278-289, doi: 10.1109/4235.687888.

[2] Li, W.D., Ong, S.K., Nee, A.Y.C. (2002). Hybrid genetic algorithm and simulated annealing approach for the optimization of process plans for prismatic parts, International Journal of Production Research, Vol. 40, No. 8, 18991922, doi: $10.1080 / 00207540110119991$.

[3] Ma, G.H., Zhang, Y.F., Nee, A.Y.C. (2000). A simulated annealing-based optimization algorithm for process planning, International Journal of Production Research, Vol. 38, No. 12, 2671-2687, doi: 10.1080/002075400411420.

[4] Li, W.D., Ong, S.K., Nee, A.Y.C. (2004). Optimization of process plans using a constraint-based tabu search approach, International Journal of Production Research, Vol. 42, No. 10, 1955-1985, doi: 10.1080/00207540 310001652897.

[5] Lian, K.L., Zhang, C.Y., Shao, X.Y., Zeng, Y.H. (2011). A multi-dimensional tabu search algorithm for the optimization of process planning, Science China Technological Sciences, Vol. 54, No. 12, 3211-3219, doi: 10.1007/s11431011-4594-7.

[6] Liu, X.-J., Yi, H., Ni, Z.-H. (2013). Application of ant colony optimization algorithm in process planning optimization, Journal of Intelligent Manufacturing, Vol. 24, No. 1, 1-13, doi: 10.1007/s10845-010-0407-2.

[7] Wang, J.F., Wu, X., Fan, X. (2015). A two-stage ant colony optimization approach based on a directed graph for process planning, The International Journal of Advanced Manufacturing Technology, Vol. 80, No. 5, 839-850, doi: 10.1007/s00170-015-7065-7.

[8] Hu, Q., Qiao, L., Peng, G. (2015). An ant colony approach to operation sequencing optimization in process planning, (In press), Proceedings of the Institution of Mechanical Engineers, Part B: Journal of Engineering Manufacture, doi: $10.1177 / 0954405415616786$. 
[9] Guo, Y.W., Mileham, A.R., Owen, G.W., Li, W.D. (2006). Operation sequencing optimization using a particle swarm optimization approach, Proceedings of the Institution of Mechanical Engineers, Part B: Journal of Engineering Manufacture, Vol. 220, No. 12, 1945-1958.

[10] Kafashi, S., Shakeri, M., Abedini, V. (2012). Automated setup planning in CAPP: a modified particle swarm optimisation-based approach, International Journal of Production Research, Vol. 50, No. 15, 4127-4140, doi: 10.1080/00207543.2011.592157.

[11] Wang, Y.F., Zhang, Y.F., Fuh, J.Y.H. (2012). A hybrid particle swarm based method for process planning optimisation, International Journal of Production Research, Vol. 50, No. 1, 277-292, doi: 10.1080/00207543.2011.571459.

[12] Li, X., Gao, L., Wen, X. (2013). Application of an efficient modified particle swarm optimization algorithm for process planning, The International Journal of Advanced Manufacturing Technology, Vol. 67, No. 5, 1355-1369, doi: 10.1007/s00170-012-4572-7.

[13] Miljković, Z., Petrović, M. (2016). Application of modified multi-objective particle swarm optimisation algorithm for flexible process planning problem, (In press), International Journal of Computer Integrated Manufacturing, doi: 10.1080/0951192X.2016.1145804.

[14] Wen, X.-Y., Li, X.-Y., Gao, L., Sang, H.-Y. (2014). Honey bees mating optimization algorithm for process planning problem, Journal of Intelligent Manufacturing, Vol. 25, No. 3, 459-472, doi: 10.1007/s10845-012-0696-8.

[15] Huang, W., Hu, Y., Cai, L. (2012). An effective hybrid graph and genetic algorithm approach to process planning optimization for prismatic parts, The International Journal of Advanced Manufacturing Technology, Vol. 62, No. 9, 1219-1232, doi: 10.1007/s00170-011-3870-9.

[16] Kennedy, J., Eberhart, R. (1995). Particle swarm optimization, In: Proceedings of the 1995 IEEE International Conference on Neural Networks, Perth, WA, IEEE Press, Vol. 4, 1942-1948, doi: 10.1109/icnn.1995.488968.

[17] Hrelja, M., Klancnik, S., Irgolic, T., Paulic, M., Jurkovic, Z., Balic, J., Brezocnik, M. (2014). Particle swarm optimization approach for modelling a turning process, Advances in Production Engineering \& Management, Vol. 9, No. 1, 21-30, doi: 10.14743/apem2014.1.173.

[18] Wang, Y., Liu, J.H. (2010). Chaotic particle swarm optimization for assembly sequence planning, Robotics and Computer-Integrated Manufacturing, Vol. 26, No. 2, 212-222, doi: 10.1016/j.rcim.2009.05.003.

[19] Malik, J., Mishra, R., Singh, I. (2011). PSO-ANN approach for estimating drilling induced damage in CFRP laminates, Advances in Production Engineering \& Management, Vol. 6, No. 2, 95-104.

[20] Xu, X., Wang, L., Newman, S.T. (2011). Computer-aided process planning - A critical review of recent developments and future trends, International Journal of Computer Integrated Manufacturing, Vol. 24, No. 1, 1-31, doi: $10.1080 / 0951192 X .2010 .518632$.

[21] Dai, M., Tang, D., Xu, Y., Li, W. (2015). Energy-aware integrated process planning and scheduling for job shops, Proceedings of the Institution of Mechanical Engineers, Part B: Journal of Engineering Manufacture, Vol. 229, No. 1, 13-26, doi: 10.1177/0954405414553069.

[22] Wang, J., Fan, X., Zhang, C., Wan, S. (2014). A graph-based ant colony optimization approach for integrated process planning and scheduling, Chinese Journal of Chemical Engineering, Vol. 22, No. 7, 748-753, doi: 10.1016/i.cjche.2014.05.011. 
APEM

\title{
Simulacijski pristop za reševanje načrtovanja procesov $z$ uporabo modificiranega algoritma rojev delcev
}

\author{
Wang, J.F. ${ }^{a,}{ }^{*}$, Kang, W.L. , Zhao, J.L. ${ }^{a}$, Chu, K.Y. ${ }^{a}$ \\ ${ }^{\mathrm{a}}$ Department of Mechanical Engineering, North China Electric Power University, Baoding, China
}

\section{POVZETEK}

Zaradi zapletenosti in raznolikosti realnih proizvodnih pogojev so računalniško podprti sistemi za načrtovanje procesov (angl. CAPP) postali v sodobni proizvodnji zelo pomembni. Načrtovanje procesov vključuje dve opravili, in sicer določitev operacij in vrstni red njihovega izvajanja. $V$ delu smo za optimizacijo proizvodnih navodil, pridobljenih iz zapletenih kosov, modificirali tradicionalni algoritem rojev delcev (angl. PSO). Vpeljali smo učinkovito kodiranje in dekodiranje populacije rešitev in tako dosegli prilagoditev problema načrtovanja procesov postopku PSO. Da bi se izognili zdrsom v lokalne optimalne rešitve smo ustrezno nastavili tudi parametre za nadzor iteracij. V običajni PSO algoritem smo vključili tudi dodatne operatorje. Da bi potrdili učinkovitost predlaganega pristopa smo izvedli več simulacijskih eksperimentov. Rezultati so pokazali, da predlagan pristop omogoča učinkovitejše reševanje problemov načrtovanja procesov kot preostali postopki.

(c) 2016 PEI, University of Maribor. All rights reserved.

\section{PODATKI O ČLANKU}

Ključne besede:

Načrtovanje procesov

Določitev operacij

Vrstni red operacij

Optimizacija z rojem delcev

Dodatni operatorji

*Kontaktna oseba: wjf266@163.com (Wang, J.F.)

Zgodovina članka:

Prejet 29. januarja 2016

Popravljen 27. marca 2016

Sprejet 1. maja 2016 